\title{
7 Peripherien in Bewegung: EIN FORSCHUNGSÜBERBLICK
}

\author{
Susanne FREINGRUBER, Institut für Geographie und Regional- \\ forschung, Universität Wien
}

\section{INHALT}

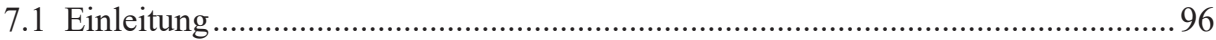

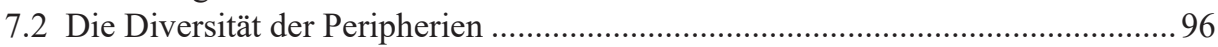

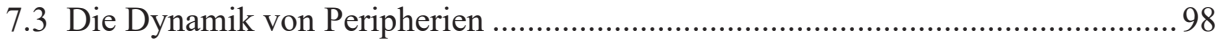

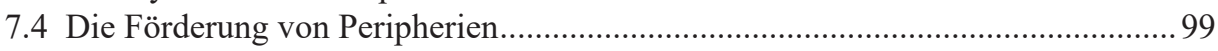

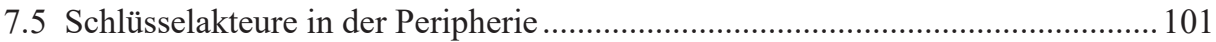

7.5.1 Innovative Unternehmen in der Peripherie ........................................................ 101

7.5.2 Weitere potenzielle Schlüsselakteure der Regionalentwicklung........................ 103

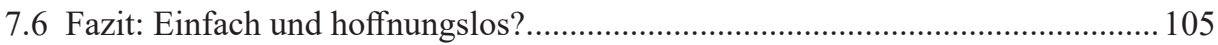

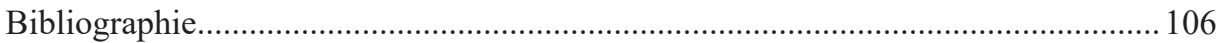

Peripherien werden derzeit oft als homogen betrachtet. Doch Peripherien sind diverser, als lange Zeit angenommen. Das Verständnis von „Peripherie“ muss daher überdacht werden, denn ein vereinfachender Peripherie-Begriff kann weder die Realität zufriedenstellend erklären, noch sind allgemeine Lösungsansätze für Peripherien besonders vielversprechend. Dieser Literaturüberblick dient folglich dazu, die veränderte Auffassung von Peripherien in der jüngeren Forschung näher zu beleuchten, die stärker prozessorientiert ist und dadurch ein höheres Erklärungspotenzial für die Diversität und unterschiedlichen Dynamiken von Peripherien aufweist. Auf dieser Grundlage zeigt sich die Notwendigkeit für individuelle Entwicklungsstrategien für Regionen, die verstärkt unter dem Aspekt von regionalen Schlüsselakteuren diskutiert werden. 


\subsection{Einleitung}

Räumliche Ungleichheiten bestehen zwischen und innerhalb von Staaten, Ländern, Städten und Regionen und sind dadurch allgegenwärtig in unserer Zeit (KüHN \& WECK 2013; IAMMARINO et.al 2018). Vor allem in den letzten beiden Jahrzehnten nahmen die regionalen Ungleichheiten in der EU erheblich zu (IAMMARINo et.al 2018). Verstärkt diskutiert werden regionale Disparitäten auch in Österreich bereits seit den 1980er Jahren (DAX \& OEDL-WIESER 2010).

Betrachtet man die räumlich ungleiche Entwicklung von Regionen näher, so wird man unweigerlich mit den Begriffen Zentrum und Peripherie konfrontiert (LANG 2016), die zumeist als Gegensatz zueinander angeführt werden (EDER 2019), aber stets in Beziehung zueinanderstehen (HeINTEL 1999). Während den Zentren eine gute Transportinfrastruktur, ein wachsender Arbeitsmarkt, eine zunehmende Anzahl an Jobs, das Vorhandensein von großen Unternehmen und der Mittelpunkt von politischen Entscheidungen zugesprochen wird, stellen Peripherien im klassischen Verständnis Räume dar, die eine schlechte Anbindung aufweisen und von Bevölkerungsrückgang, Jobverlusten, geringer Wissensintensität und wenig politischen Einfluss gekennzeichnet sind (EDER 2019). Dadurch erfahren sie eine wirtschaftliche und soziale Benachteiligung im Gegensatz zu den Zentren. Innerhalb eines Landes bedeutet dies nicht selten, dass Städte auf der einen Seite als Zentren bezeichnet werden, der ländliche Raum auf der anderen Seite als Peripherie (LeSER et.al 2011). Überwiegend wird die Definition von Peripherien auch mit der Bestimmung der Erreichbarkeit von Angeboten in den Zentren gekoppelt. Die Distanz zu einem Zentrum ist daher dominierend im Begriffsverständnis von Peripherien, das in der Regel räumlich erfolgt (LANG 2016).

Lage, Siedlungsdichte und Distanz, bzw. Erreichbarkeit in Beziehung zu einem Zentrum scheinen in diesem Zusammenhang entscheidend zur Identifizierung von Peripherien. Diese räumliche Auffassung von Peripherien kann weitgehend problemlos über statistische Variablen bestimmt werden (LANG 2016), sie unterstreicht jedoch auch die Wahrnehmung von Peripherien als räumlich-statischen Zustand (KÜHN \& WeCK 2013). Dadurch erscheint es recht unwahrscheinlich, dass sich Peripherien auch positiv entwickeln können. Doch ist es wirklich so einfach? Und hoffnungslos?

\subsection{Die Diversität der Peripherien}

Ein Ausflug aufs Land. Ein Stopp im Waldviertel. Über 1 Stunde von Wien entfernt. Ein ländlicher Raum, relativ dünn besiedelt. Eine periphere Region? Wie bewertet die aktuelle Forschung generell solche Rückschlüsse? Braucht es nicht mehr als diese Angaben, um periphere Regionen bestimmen zu können? 
Zunächst weist EDER 2019 darauf hin, dass ländliche Räume nicht pauschal mit dem Begriff Peripherie gleichzusetzen sind, denn auch ländliche Räume sind divers und können unterschiedlich dynamisch sein. Eine allgemeingültige Definition von ländlichen Räumen und ebenso von peripheren ländlichen Räumen zu formulieren gestaltet sich daher mehr als schwierig und würde die Diversität dieser Räume vernachlässigen (MAIER 2008). Auch warnt HeINTEL 1999 vor einer eindimensionalen Betrachtung einer einzigen Facette, wie die geographische Distanz zu einem Zentrum, um einen Raum der Peripherie zuzuordnen, da es sich um ein viel komplexeres Themengebiet handelt, als es oftmals durch alteingesessene Assoziationen erkennbar wird. MAIER 2008 sieht die Wirtschafts- und Siedlungsstrukturen, sowie soziokulturelle Merkmale bei der ausschließlichen Betrachtung von Dichte und Erreichbarkeit vernachlässigt und betont gerade deren Bedeutung für eine zufriedenstellende Charakterisierung von Räumen, ihren Potenzialen und Problemen. KüHN \& WeCK 2013 sehen ebenso die Notwendigkeit die räumlichen Merkmale um weitere, wirtschaftliche, soziale und politische, Indikatoren zu ergänzen, um Peripherien definieren zu können.

Eine mögliche differenziertere Betrachtung wird auch von EDER 2019 vorgeschlagen, der sowohl die geographische Dimension, als auch die demographische und ökonomische Dimension in Kombination mit Wissensbasen ${ }^{1}$ in seine Analyse miteinbezieht. Die Berücksichtigung der vielseitigen unterschiedlichen Indikatoren ergeben letztlich eine große Diversität von Peripherien, aber auch von Zentren, und machen deutlich, dass ländliche Regionen mitunter geographisch peripher, aber dennoch demographisch und/oder ökonomisch zentral sein und gelegentlich starke Ausprägungen der Wissensbasen aufweisen können. Das eingangs gezeichnete klassische Verständnis von Zentren und Peripherien entspricht demnach lediglich den zwei Extremen zwischen denen es vielerlei Abstufungen gibt (EDER 2019). In der aktuellen Forschung zeigt sich daher, dass es mehr bedarf, als einer einfachen Schlussfolgerung von einem ländlichen, großstadt- und verdichtungsfernen Raum auf eine Peripherie, wenngleich oftmals nicht vorhandenes Datenmaterial eine differenziertere Betrachtung erschwert (EDER 2019; GIFFINGER \& KRAMER 2012).

EDER 2019 berücksichtigt sowohl die analytische, als auch die synthetische Wissensbasis. Laut AsHEIM 2007 findet sich die analytische Basis vor allem in wissensintensiven Branchen. F\&E Abteilungen und die Zusammenarbeit mit Universitäten ermöglichen radikale Innovationen, Neugründungen von Firmen und Spin-offs. Die synthetische Basis ist eher in der industriellen Produktion relevant und beruht auf der Anpassung oder Neukombination von vorhandenem Wissen. Die Ausbildung am Arbeitsplatz und Erfahrung sind hierfür entscheidend. 


\subsection{Die Dynamik von Peripherien}

Ein Blick in die Tageszeitung. 40 Prozent der Gemeinden in Österreich wird prophezeit, zu schrumpfen (LÖFFLER 2019). Eine andere Ausgabe der Zeitung. Eine ländliche Gemeinde im Innviertel, Munderfing. 1:30 Stunden von Linz entfernt. Die Gemeinde wächst. Die Geburtenfälle übersteigen seit kurzem die Todesfälle. Neue Unternehmen siedelten sich an (LÖFFLER 2018). Wie lassen sich diese Dynamiken erklären?

Ein Festhalten an räumlich-geographischen Merkmalen zur Definition von Peripherien ließe einem zu dem Schluss kommen, dass Peripherien eben Peripherien sind und solche auch für eine lange Zeit bleiben. Dieser Beitrag wäre in diesem Fall an diesem Punkt zu Ende, da sich als Peripherien identifizierte Regionen mit ihrem Schicksal schlichtweg abzufinden hätten und unter dieser Betrachtungsweise weitere Überlegungen demzufolge unnötig wären. Denn befindet sich eine Region in einer geographisch peripheren Lage, so wird sich dies nicht in überschaubarer Zeit verändern können (EDER 2019; KÜHN 2015). Ländliche Regionen, die sich trotz ihrer geographischen Lage in den letzten Jahren zunehmend positiv entwickeln und wachsen, machen jedoch ein Umdenken notwendig, um diese Situation erklären zu können (KüHN \& WECK 2013).

Um der Dynamik gerecht zu werden, wird nun in der räumlichen Forschung das traditionelle Verständnis von Peripherien ergänzt (KüHN 2015). Dies bedeutet, dass gegenwärtig der Prozess zur Entstehung von Peripherien diskutiert und in diesem Zusammenhang der Begriff der Peripherisierung ins Spiel gebracht wird, der im Vergleich zum Begriff Peripherie deutlicher auf die Prozesshaftigkeit hinweisen soll. Nicht mehr räumlich-statische Gegebenheiten stehen im Fokus, sondern vermehrt sozial-räumliche Prozesse „,in denen Peripherien produziert und reproduziert werden“ (KÜHN \& WeCK 2013, p. 24).

KÜHN \& WeCK 2013 schlagen vor hierbei zwischen vier Dimensionen zu unterscheiden. Zunächst dem Prozess der Abwanderung, der über längere Zeit mit einer Schwächung der Innovationsfähigkeit, sowie der Beschränkung von Lebensqualität durch die Reduzierung des lokalen Versorgungsangebotes und dem Verlust von Bildungs- sowie Arbeitsplatzchancen einhergeht. Weitere Dimensionen stellen der Prozess der Abhängigkeit von den Entscheidungen in den Zentren dar, sowie der Prozess der Abkopplung von Innovationsdynamiken, oder der technischen und sozialen Infrastruktur. Und letztlich die vierte Dimension, der Prozess der Stigmatisierung, der sowohl negative Fremd- als auch Selbstbilder umfasst, die eine Abwärtsspirale verschärfen können (KÜHN \& WECK 2013). Dass letztgenannter Prozess nicht zu unterschätzen ist, diskutiert RodRíGuez-Pose 2018 ausführlich, wenn er von der „Rache der Orte, die nicht zählen" schreibt. Er weist darauf hin, dass der ansteigende Populismus nicht vom Himmel gefallen ist, sondern eine Reaktion der Zurückgelassenen auf die Politik der Zentren ist. Er erklärt dadurch u.a. die Entscheidung zum Brexit, wo haupt- 
sächlich Wahlberechtigten außerhalb der Zentren für das Verlassen der EU stimmten, oder die knappe Entscheidung bei der österreichischen Bundespräsidentenwahl. Auch IAMMARINo et.al 2018 halten die Teile der Bevölkerung anfällig für Populismus, die sich im Stich gelassen fühlen.

Die Analyse von ländlichen Räumen bezieht sich hinsichtlich der Regionalentwicklung jedoch meist auf die ökonomische Performance im Vergleich zum Durchschnitt des Landes, oder der Städte (DAX \& OEDL-WIESER 2010). BürChER et.al 2015 erkennen, dass die Unterschiede zwischen unterschiedlich dynamischen Peripherien noch unzureichend erforscht sind und bieten die relationale Perspektive als einen Ansatz, der ihrer Meinung nach großes Erklärungspotenzial für diese Unterschiede hat. BÜRCHER et.al 2015 betonen hierzu die Bedeutung der regionalen und überregionalen Vernetzungen. Auch KÜHN \& WeCK 2013 identifizieren eine wachsende Bedeutung von weichen Standortfaktoren, wie Netzwerken zwischen Wirtschaft, Verwaltung, Politik sowie Regional- und Projektmanagement, um die Dynamik von Regionen erfassen zu können. Diese Interaktionen sind veränderbar und verdeutlichen erneut die zunehmende Erfordernis Peripherien über statische Parameter hinaus weiterzudenken.

KÜHN 2015 konstatiert dem Konzept der Peripherisierung dennoch eine gewisse Unschärfe, da es unterschiedlich interpretiert werden kann und schwer zu prüfen ist. Zentral sieht er jedoch 5 wesentliche Punkte: (1) die Verbindung mit dem Begriff Zentralisierung im sozial-räumlichen System, (2) die Prozesshaftigkeit des Konzepts, die den dynamischen Aufstieg, oder Niedergang von Räumen erfassen kann, (3) die Multidimensionalität, (4) die Beachtung verschiedener Maßstabsebenen und (5) die Bedeutung der zeitlichen Komponente, die langfristig zu einer De-Peripherisierung oder Re-Zentralisierung führen kann. Diese Aspekte in den Diskurs einzubeziehen, erscheinen entscheidend bei einer zeitgemäßen Auseinandersetzung mit Peripherien (KüHN 2015).

\subsection{Die Förderung von Peripherien}

Erlaubt man den Gedanken, dass sich Peripherien verändern können, so stellt sich die Frage, wie eine Entwicklung in peripheren Räumen gefördert werden kann, um die Prozesse der Abwanderung, Abkopplung, Abhängigkeit und Stigmatisierung für periphere Räume abzubremsen, oder gar umzukehren. Hierzu gibt es mehrere Ansätze.

Der neoklassische Zugang etwa besagt, dass es keinerlei Eingriffe brauche, die Ungleichheiten zwischen Zentrum und Peripherie würden sich von selbst regeln (IAMMARINO et.al 2018), da sich die Entwicklungsunterschiede „langfristig über eine den Märkten innewohnende Gleichgewichtstendenz angleichen“ würden (KÜHN \& WECK 2013, p. 25). Vor noch wenigen Jahren wurden der zunehmenden Mobilität, sowie der Digitalisierung und Globalisierung zugesprochen, dass sie zu einem Ausgleich zwischen Zentrum und Peripherie führen können. So mancher sieht den Erfolg jedoch nicht als 
ausreichend gegeben (SILL 2019). Mancherorts gelangt man heutzutage erneut zu der Ansicht, dass ein Eingriff nicht erforderlich wäre. Nicht, weil davon ausgegangen wird, dass es auch ohne Maßnahmen zu einem Ausgleich kommen würde, sondern weil gewissermaßen das Gefühl der Aussichtslosigkeit überhand zu nehmen scheint. Man könne nicht alle Orte retten, wie manche Wirtschaftsforscher verlautbaren (LÖFFLER 2019).

Der Fokus der Politik für den ländlichen Raum liegt laut GASSER 2013 anhaltend auf Landwirtschaftspolitik, obwohl die Zahl der Bauern im ländlichen Raum mittlerweile verschwindend gering ist. Politische Entscheidungsträger forcieren aber auch oftmals die physische Anbindung an Zentren zu verbessern, wie sich an dem konkreten Beispiel der aktuell in Österreich propagierten Waldviertelautobahn zeigen lässt (LÖFFLER 2018). Obwohl die räumliche Entfernung zu Wirtschaftszentren eine tatsächliche Herausforderung für periphere Räume darstellt (DAX \& OEDL-WIESER 2010), lehnen IAMMARINO et.al 2018 diese Infrastrukturmaßnahme eher ab, denn eine physische Anbindung führe nicht wie angenommen zu mehr Arbeitsplätzen, steigender Produktivität und Wirtschaftswachstum im peripheren Raum, sondern verstärke im Gegenteil meist noch mehr die Zentralisierung und reduziert weiter das lokale Angebot. PIKE et.al 2017 untersuchten die unterschiedlichen Konzepte zu Lokal- und Regionalentwicklung ab dem 19. Jahrhundert und stufen diese Vorschläge von Politikern als sehr typisch ein. „Obwohl der räumliche Keynesianismus zurückgeht", schreiben sie, „bleiben Investitionen in die physische Infrastruktur [...] zentral für politische Entscheidungsträger" (PIKE et.al 2017, p. 53). Diese top-down Maßnahmen werden in der Literatur zur Regionalentwicklung jedoch grundsätzlich eher kritisch gesehen. CHRISTMANN 2014 beschreibt, wie nicht nachhaltig top-down Bemühungen, u.a. von der EU, oftmals sind. Diese Ansätze würden meistens stoppen, sobald die Förderung endet und reflektieren selten die regionalen Bedingungen. Die Autorin erklärt, dass es wesentlich nachhaltiger wäre, wenn sich regionale Akteure aktiv beteiligen würden (CHRISTMANN 2014).

In der Regionalwissenschaft kam es bereits in den letzten Jahrzehnten zu einem stärkeren Fokus auf eine endogene Entwicklung unter Berücksichtigung von regionalen Bedingungen (GIFFINGER \& KRAMER 2012). Dass die Lösung aus der Region kommen muss, wenn sie wirken soll, sehen daher auch viele Autoren, die die Bedeutung von endogenen Entwicklungsstrategien betonen (vgl. z.B. MAYER 2016, EDER 2019, Baumgartner et.al 2013, Sotarauta 2010). Dieser Ansatz deckt sich mit der zuvor erörterten Diversität von (peripheren) Räumen. Da für unterschiedliche Räume nicht, wie IAMMARINO et.al 2018 es nennen, orts-neutrale Strategien die Lösung von räumlich ungleicher Entwicklung sein können. Eine Pauschallösung kann es daher nicht geben und gerade deshalb gestaltet sich die Förderung der Entwicklung von Peripherien sehr komplex.

Jedoch sehen BAUMGARTNER et.al 2013, die die verschiedenen Typen von Entrepreneurship in ländlichen Regionen beschreiben, „die Förderung von Innovationen, die Mobilisierung von Sozialkapital und die Förderung von institutionellem Wandel als Schlüsselherausforderungen für die erfolgreiche Implementierung jeder endogenen Wachstumsstrategie“" (BAUMGARTNER et.al 2013, p. 1101). MERKL 2007 merkt an, 
dass Innovation die „wichtigste Voraussetzung für Wettbewerbsstärke, Beschäftigung, soziale Sicherheit, Wohlstand und Lebensqualität“ ist (MerkL 2007, p. 78). Sozialkapital wird von BACHINGER 2012 als ein Netzwerk von Vereinen, Aktivitäten oder Beziehungen beschrieben, die für eine Gemeinschaft bedeutend sind und deren wesentlicher Bestandteil ein großes Vertrauen untereinander ist. BAUMGaRTNER et.al 2013 sehen den institutionellen Wandel als eine fortlaufende Anpassung von Beschränkungen, um Organisationen und Regionen vor negativen „lock-ins“ zu bewahren und dadurch eine Umwelt zu schaffen, die Chancen auf eine Weiterentwicklung ermöglicht.

\subsection{Schlüsselakteure in der Peripherie}

Peripherien können sich verändern. Die Lösung ihrer Probleme soll aus der Region kommen. Regionale Akteure sollen sich aktiv beteiligen und Innovationen, Sozialkapital und institutionellen Wandel hervorbringen. Doch welche Akteure haben das Potenzial dies zu bewerkstelligen?

Durch die Auffassung von Peripherisierung als Prozess zur Entstehung von Peripherien rücken nun auch zunehmend Akteure der Regionalentwicklung in den Mittelpunkt der Betrachtung, die einen positiven Einfluss auf die Entwicklung von strukturschwächeren Regionen haben können (KüHN \& WECK 2013). Entrepreneurship ist längst zum Gegenstand von wissenschaftlichen Debatten zur Entwicklung von peripheren, ländlichen Räumen geworden. Die Konzepte erscheinen gleichsam vielfältig, wie unscharf. Ein möglicher Grund dafür könnte darin liegen, dass sich aktuell viele Disziplinen dem Themengebiet annähern und es deshalb bereits an einheitlichen Definitionen scheitert. Neben dem klassischen Verständnis von Entrepreneurs als Unternehmer, die Innovationen hervorbringen, finden auch zum Beispiel jene Beachtung für die die Schaffung von sozialem Wert im Vordergrund stehen, oder die einen Wandel der Strukturen bewirken können (BAUMGARTNER et.al 2013).

Es folgt nun einerseits ein kurzer Blick auf innovative Unternehmen, um zu verstehen, wie es funktionieren kann erfolgreich in peripheren Regionen zu sein und andererseits auf eine kleine Auswahl an weiteren regionalen Schlüsselakteuren, die durchaus auch in der Lage sein können Impulse für eine positive Veränderung in einer Region zu setzen.

\subsubsection{Innovative Unternehmen in der Peripherie}

Die Wissenschaft konzentrierte sich bislang bei Innovationsprozessen auf die Forschung in den Zentren. Peripherien werden hingegen selten mit Innovationen in Verbindung gebracht. Fehlende Bildungsorganisationen, die zu geringe Anzahl an 
Unternehmen, um Clustering- oder Agglomerationseffekte zu erzielen, sowie ein niedriger Grad der Vernetzung der hauptsächlichen KMUs zählen zu den häufigsten Argumenten, die gegen die Bedeutung von Peripherien in Verbindung mit Innovationen sprechen (BÜRCHER et.al 2015). Als größte Schwierigkeit für Unternehmen in der österreichischen Peripherie zählt zudem das begrenzte Angebot von qualifizierten Arbeitskräften. Aber auch, wenn Unternehmen in der Peripherie grundsätzlich verschiedene Nachteile gegenüber Zentren zugesprochen werden, die es ihnen erschweren innovativ sein zu können und sie tatsächlich nicht in Massen vertreten sind, gibt es sie dennoch, die innovativen Unternehmen in der Peripherie (EDER \& TRIPPL 2019).

EDER \& TRIPPL 2019 untersuchten mehrere innovative Unternehmen unterschiedlicher Größe in der Peripherie in Österreich und identifizierten verschiedene Strategien, die diese Unternehmen anwenden, um innovativ sein zu können. Kompensationsstrategien, wie zum Beispiel die Stärkung der internen Kompetenz durch Aus- und Weiterbildungen der Arbeitskräfte, die Zusammenarbeit mit Universitäten, oder die Inanspruchnahme von modernen Kommunikationstechnologien, werden genutzt, um den Herausforderungen eines Standortes in der Peripherie erfolgreich zu begegnen. Nachteile werden ebenso in Hinblick auf das begrenzte Fachkräfteangebot versucht zu kompensieren. Dies erfolgt eventuell durch eine zusätzliche Niederlassung in einem Zentrum, um von diesem Arbeitsmarkt profitieren zu können. Wenn Unternehmen das Gefühl haben mehr für die Anwerbung und das Halten von Arbeitskräften unternehmen zu müssen, als Firmen in den Zentren, so kann auch das ,employer branding“ zu den firmeninternen Strategien zählen (EDER \& TRIPPL 2019).

Die Bedeutung von angeworbenen Fachkräften aus anderen Firmen, vor allem aus jenen in Städten, sehen MAYER et.al 2016 in dem Wissen, das diese aus ihrer früheren Tätigkeit mitbringen und mit dem neuen Unternehmen verknüpfen können. Sie verfügen darüber hinaus über Netzwerke, die, wenn sie sie aktiv weiterentwickeln, gute Chancen bringen, um Benachteiligungen aufgrund des peripheren Standortes zu verringern. Zudem wird diesen Fachkräften zugeschrieben Marktbedürfnisse und Möglichkeiten besser erkennen zu können (MAYER et.al 2016). BAUMGARTNER et.al 2013 heben bei angeworbenen Managern ebenso deren großen Erfahrungswert und ihren Blick von außen hervor. Zudem sehen sie die von ihnen gemanagten Unternehmen als innovations- und wachstumsorientierter als jene von lokalen. Auch DAX \& OEDL-WieSER 2010 betonen generell das kreative Potenzial, das von externen Personen eingebracht werden kann, wodurch Impulse für eine positive Entwicklung gesetzt werden können.

Dass ein Standort in der Peripherie ausschließlich Nachteile für die Unternehmen bringt, die jene mit einem Set an Strategien überwinden müssen, trifft entgegen üblicher Meinungen nicht zu. Denn ein peripherer Standort birgt durchaus auch Potenziale, die Unternehmen mittels Verwertungsstrategien für sich nutzen können. Vorteile sehen einige Unternehmen in der großen Loyalität von Mitarbeitern, der geringen Konkurrenz, wodurch sowohl die Unterstützung von politischen Entscheidungsträgern, als auch der Einfluss auf Lehrpläne von nahegelegenen Ausbildungseinrichtungen möglich sind, 
sowie in der Attraktivität des Landschaftsbildes und in finanziellen Anreizen. Welche Kombination von Strategien ein Unternehmen letztlich anwendet ist individuell und je nach Wahrnehmung der Herausforderungen für ein Unternehmen verschieden. Festzuhalten ist, dass es auch hier keine einheitlichen Strategien gibt (EDER \& TRIPPL 2019).

Auch wenn es letztlich nicht viele innovative Unternehmen in der Peripherie geben mag, es zeigt sich dennoch, dass es sich lohnt sie in die Forschung miteinzubeziehen, um von ihnen zu lernen. Denn nicht zuletzt können innovative, wachstumsorientierte Unternehmer auch als potenzielle Vorbilder in einer Region gesehen werden (BAUMGARTNER et.al 2013).

\subsubsection{Weitere potenzielle Schlüsselakteure der Regionalentwicklung}

Wer sich mit einer Gemeinde auseinandersetzt, so möchte ich unterstellen, wird unweigerlich auch an das lokale „Oberhaupt“ eines Ortes denken, den Bürgermeister. Die Bedeutung der Bürgermeister für die positive Entwicklung einer Gemeinde ist in der Literatur allerdings umstritten. Während Autoren wie BAUMGARTNER et.al 2013 aufzeigen, dass initiative Bürgermeister das Kernstück des ländlichen community Entrepreneurships sein können, da sie die Fähigkeiten haben öffentliche und private Ressourcen sowie einen großen Teil der lokalen Bevölkerung zusammenzuführen, schreiben hingegen Autoren wie PIKE et.al 2017 den Bürgermeistern keine bedeutende Rolle für eine gute soziale und wirtschaftliche Entwicklung zu, weil es dafür kaum begründete Beweise gäbe. Beide Ansichten haben wohl ihre Berechtigung, denn obwohl es schlüssig erscheint, dass die Bürgermeister die Entwicklung ihrer Gemeinde beeinflussen können, zeigt die Realität häufig eine Haltung, die mit Resignation vergleichbar wäre. Dennoch existieren vereinzelt Bürgermeister, die sehr wohl Visionen haben, eventuell Betriebswohnungen ermöglichen, sich um die Ansiedlung einer FH bemühen oder Glasfaserkabel für alle forcieren wollen, sowie das große Ziel haben, das örtliche Gasthaus wieder aufzusperren, oder schlichtweg Unternehmen eine Ansiedlung auf Gemeindeboden erleichtern (LÖFFLER 2018).

SOTARAUTA 2010 bringt für engagierte Akteure die Bezeichnung Regional Development Officer (RDO) ins Spiel. Das sind strategische Akteure (im Fall des Bürgermeister Generalisten), die eine gute Wahrnehmung für Trends und ihre Bedeutung haben und durch viele Interessen manövrieren müssen. Sie steigern das Bewusstsein für Neues, mobilisieren, rahmen, koordinieren und organisieren Prozesse. SotaRAUTA 2010 erklärt darüber hinaus, dass es viele Formen von RDOs gibt, es aber immer eine entwicklungs- und wachstumsorientierte Person ist, die auf irgendeine Art und Weise die Region entwickeln will. Diese recht weit gefasste Definition erlaubt es die grundsätzliche Bedeutung eines Bürgermeisteramtes daher tatsächlich als wesentlich für eine positive Entwicklung einer Gemeinde einzustufen, da man von diesen Akteuren einen Wandlungswillen, ein Gespür für die Bedürfnisse der Gemeinde und Verhandlungsgeschick erwarten würde. 
Dennoch zeigen genügend Negativbeispiele, dass Bürgermeister öfters an ihre Grenzen stoßen, oder die Augen zu verschließen scheinen und keine Schlüsselrolle verkörpern können. Gasthäuser bleiben zu, Innovationen bleiben aus, die Abwärtsspirale setzt sich fort (GASSER 2013, LÖFFLER 2019). Wenn es in einer peripheren Region eventuell innovative Ideen geben sollte, so fehlt es mitunter an treibenden Kräften, Fachwissen und Geldmitteln (CHRISTMAnN 2014). Auch die Bürokratie hinter Förderungen setzt vielen zu (GASSER 2013). CHRISTMANN 2014 sieht eine mögliche Lösung in sozialen Entrepreneurs als Brückenbauer zwischen top-down und bottom-up Initiativen. Soziale Entrepreneurs definiert sie als Visionäre, die entweder innovative Lösungen für soziale Probleme selbst entwickeln und einführen, oder anderen Akteuren in der Region Hilfe zur Selbsthilfe offerieren. Sie bieten ihr Expertenwissen, organisieren entweder neue Ausbildungsprogramme, beraten oder coachen, sie stellen hilfreiche Kontakte zur Verfügung und Informationen zu sozialen Netzwerktechniken, sind bei finanziellen Angelegenheiten behilflich und setzen so strategische bottom-up Prozesse in Gang, die es ohne sie nicht gegeben hätte, oder treiben einen weiteren bottom-up Prozess voran (Christmann 2014).

BAUMGARTNER et.al 2013 sehen social Entrepreneurship als einen Driver für Sozialkapital, das von lokalen Initiativen geschaffen und verstärkt wird indem öffentliche Güter und Services, die es nicht mehr im öffentlichen Service gab, gestaltet werden. Zudem fördern sie das gegenseitige Vertrauen in einer Gemeinschaft. Anders als Christmann 2014 betonen Baumgartner et.al 2013 nicht explizit die Innovativität der Lösungen. BAUMGARTNER et.al 2013 sehen aber auch die Notwendigkeit, dass jene Akteure besondere Fähigkeiten, wie koordinieren, führen und managen, aufweisen müssen, um ein positives Ergebnis erzielen zu können. Dieses liegt -und hier sind sich die genannten Autoren einig- in der Produktion von zusätzlichem sozialem Wert für eine bestimmte Gesellschaft, wobei der Fokus nicht auf den Profit gerichtet ist (BAUMGARTNER et.al 2013, Christmann 2014). Weiterer Forschungsbedarf zu sozialen Entrepreneurs besteht gewiss. CHRISTMann 2014 konnte lediglich vier Projekte in Europa aufspüren, die von drei verschiedenen sozialen Entrepreneurs initiiert wurden.

LEICK 2017 erkennt großes Potenzial in institutionellen Entrepreneurs, die für Unternehmen eine große Hilfe darstellen können. Denn Entwicklungen, wie der demographische Wandel, werden in den kommenden Jahrzehnten zunehmend zur zentralen Herausforderung für Unternehmen, wenn das ohnehin schon eingeschränkte Arbeitskräfteangebot in peripheren Räumen weiter reduziert wird sobald ältere Fachkräfte das Pensionsalter erreicht haben. Institutionelle Entrepreneurs können zunächst das Bewusstsein für die Herausforderung bei Unternehmen wecken und in einem weiteren Schritt die Stärkung der Unternehmen in Bezug auf ihre interne Kompetenz unterstützen (LEICK 2017).

MiÖRNER \& TRIPPL 2017 beschäftigten sich weniger mit der Wandlung von Strukturen innerhalb eines Unternehmens, sondern setzten sich mit dem institutionellen und organisatorischen Wandel der Unterstützungsstrukturen für die Etablierung einer neuen Industrie auseinander. Sie zeigten verschiedene Wege auf, wie Akteure mit unterschied- 
lichem Einfluss neue Strukturen, wie eine Fachkonferenz, oder neue Ausbildungsprogramme, hervorbringen können. Während ein Wandel zunächst möglicherweise nur von einem Einzelnen begonnen wird, ist es am Ende doch ein kollektiver, multiskalarer Prozess, der zu einer erfolgreichen Umgestaltung der einstigen, ungünstigen Strukturen führt (MiöRNER \& TRIPPL 2017).

Letztlich bedarf es wohl Visionäre als Schlüsselfiguren (CHRISTMANN 2014), die die Bedürfnisse und Probleme einer Region sowie zutage tretende Möglichkeiten erkennen (BAUMGARTNER et.al 2013) und durch ihre Fähigkeiten weitere Akteure zusammenbringen (SOTARAUTA 2010), die dann im Kollektiv (MiöRnER \& TrIPPL 2017), positiven Einfluss auf die Entwicklung einer Region ausüben können.

\subsection{Fazit: Einfach und hoffnungslos?}

Es zeigt sich, dass es sich bei Peripherien keinesfalls um ein einfaches Themengebiet handelt, das kurz und knapp abgehandelt werden kann. Der Diversität von peripheren Räumen Beachtung zu schenken und über räumlich-statische Merkmale hinauszudenken erscheint als eine Notwendigkeit, um der Realität gerecht zu werden. Die verstärkte Konzentration auf den Prozess zur Entstehung von Peripherien in der wissenschaftlichen Debatte sehe ich in diesem Zusammenhang ebenso bedeutsam, da dies erneut sowohl die Diversität von peripheren Räumen betont, als auch Peripherien eine gewisse Wandlungsfähigkeit zugesteht. Die unterschiedlichen Dimensionen, die zur Erklärung von Peripherien herangezogen werden, erfordern es stets genauer hinzusehen, wie Peripherien definiert werden.

Die Komplexität der Thematik macht sich darüber hinaus in der Suche nach adäquaten Möglichkeiten zur Förderung von peripheren Regionen bemerkbar. Während top-down Ansätze als Einheitslösungen in der Literatur wenig Anklang finden, erscheinen endogene Entwicklungsmaßnahmen, die die regionalen Bedingungen reflektieren, positiver bewertet zu werden. Einfacher ist dieser Lösungsweg jedoch bestimmt nicht. Doch maßgeschneiderte Ansätze für den jeweiligen peripheren Raum zu suchen erscheint nachvollziehbar. Denn wenn es unterschiedlich dynamische Peripherien gibt, so haben diese auch unterschiedlich stark ausgeprägte Herausforderungen zu bewältigen und brauchen in einer logischen Konsequenz auch individuelle Lösungen.

Innovationen, Sozialkapital und der institutionelle Wandel erscheinen jedoch essenziell, um überhaupt eine positive Entwicklung in einer Region in Gang setzen zu können. Die notwendige Beteiligung von regionalen Akteuren bei dieser Entwicklung von innen heraus wird in der aktuellen Literatur sehr deutlich. Die häufige Resignation, Verleugnung, oder Überforderung von Lokalpolitikern kann es erforderlich machen, dass von anderen Akteuren Impulse gesetzt werden müssen. Die wissenschaftlichen Konzepte, wer diese Akteure sein könnten, sind vielfältig. Sowohl innovative Unter- 
nehmen können ihren Teil beitragen, als auch weitere Akteure, die etwas verändern wollen und dazu bereit sind sich zu engagieren.

Letztlich, so scheint es, bedarf es jedoch eines Zusammenspiels von mehreren Akteuren, um sich den meist komplexen Herausforderungen von peripheren, ländlichen Regionen erfolgreich anzunehmen. Denn Peripherien können sich wandeln und wenn sich diese Akteure finden lassen, so muss die Situation nicht zwingend hoffnungslos sein. Es sollten jedoch nicht die Hände in den Schoß gelegt, die Augen verschlossen und auf ein Wunder gewartet werden.

\section{Bibliographie}

Asheim B. (2007), Differentiated knowledge bases and varieties of regional innovation systems. Innovation: The European Journal of Social Science Research 20(3), pp. 223-241.

BACHINGER M. (2012), Stakeholder Value in Regionalentwicklungsprozessen. Eine relationale Perspektive. Wiesbaden, Gabler.

Baumgartner D., Pütz M., Seidl I. (2013), What Kind of Entrepreneurship Drives Regional Development in European Non-core Regions? A Literature Review on Empirical Entrepreneurship Research. European Planning Studies 21(8), pp. 1095-1127.

BürCher S., Habersetzer A.J., MaYer H. (2015), Entrepreneurship in peripheral regions: A relational perspective. In: MACK, E.A., QIAN H. (eds.), Geographies of Entrepreneurship. Routledge studies in human geography. New York, Routledge, pp. 143-164.

Christmann G.B. (2014), Social Entrepreneurs on the Periphery: Uncovering Emerging Pioneers of Regional Development. disP - The Planning Review 50(1), pp. 43-55.

DAX T., OedL-Wieser T. (2010), Periphere ländliche Regionen im Brennpunkt - Der ländliche Raum zwischen Dynamik und Entleerung. Online verfügbar unter $<$ https://www.bmnt. gv.at/dam/jcr:e49dea94-8d73-46b6-a2dd-254d8ee4b265/08_DAX_LR_Periphere_201 ndliche_20Regionen_TD_TOW_2010.pdf>

Eder J. (2019), Peripheralization and knowledge bases in Austria: towards a new regional typology. European Planning Studies 27(1), pp. 42-67.

EDER J., TRIPPL M. (2019), Innovation in the periphery: compensation and exploitation strategies. Online verfügbar unter <https://onlinelibrary.wiley.com/doi/epdf/10.1111/grow.12328>

Gasser F. (2013), Stadt, Land, Flucht. Online verfügbar unter <https://www.zeit.de/2013/29/ oesterreich-landflucht/komplettansicht?print>

Giffinger R., Kramer H. (2012), Kleinstädte als Wachstumsmotoren ländlich-peripherer Regionen: Das Beispiel Waldviertel. disP - The Planning Review 48(2), pp. 63-76.

Heintel M. (1999), Zentrum, Peripherie und Grenze. Alte und neue Herausforderungen in der europäischen Raumplanungspolitik. Online verfügbar unter $<\mathrm{http}: / /$ www.demokratiezentrum. org/fileadmin/media/pdf/heintel.pdf>

Iammarino S., Rodriguez-Pose A., Storper M. (2018), Regional inequality in Europe: evidence, theory and policy implications. Journal of Economic Geography 19(2), pp. 273-298.

KüHN M. (2015), Peripheralization: Theoretical concepts explaining socio-spatial inequalities. European Planning Studies 23(2), pp. 367-378. 
KüHN M., WECK S. (2013), Peripherisierung - ein Erklärungsansatz zur Entstehung von Peripherien. In: Bernt M. \& Liebmann H.(eds.), Peripherisierung, Stigmatisierung, Abhängigkeit? Deutsche Mittelstädte und ihr Umgang mit Peripherisierungsprozessen. Springer, Wiesbaden, pp. 24-46.

LANG T. (2016), Peripherie / Peripherisierung. Online verfügbar unter $<$ https://www.researchgate. net/publication/294848196_Peripherie_Peripherisierung>

LEICK B. (2017), Institutional entrepreneurs and small firms: How firm practices are being shaped in the context of demographic transformations. Journal of Small Business and Entrepreneurship 29(1), pp. 1-24.

Leser H., Egner H., Meier S., Mosimann T., Neumair S., Paesler R., Schlesinger D. (2011) ${ }^{15}$, „Diercke. Wörterbuch Geographie. Raum - Wirtschaft und Gesellschaft - Umwelt“". Braunschweig, Westermann.

LÖFfLER A. (2018), Abwanderung aus dem Dorf: Stadt, Land, Landflucht. Die Presse, 29.01.2018. Online verfügbar unter $<$ https://www.diepresse.com/5362324/abwanderung-aus-dem-dorfstadt-land-landflucht>

LÖFfLER A. (2019), Landflucht: Keiner will von sterbenden Dörfern sprechen. Die Presse, 09.04.2019. Online verfügbar unter <https:/www.diepresse.com/5609402/landfluchtkeiner-will-von-sterbenden-dorfern-sprechen>

MaIER J. (2008), Definitorische Abgrenzung peripherer ländlicher Räume und inhaltliche bzw. regionale Auswahl der Beiträge. Online verfügbar unter $<$ https://www.ssoar.info/ ssoar/bitstream/handle/document/34113/ssoar-2008-maier-Definitorische_Abgrenzung peripherer_landlicher_Raume.pdf?sequence $=1 \&$ isAllowed $=\mathrm{y} \& \ln k$ name $=$ =ssoar-2008maier-Definitorische_Ā

Mayer H., Habersetzer A., Meili R. (2016), Rural-Urban Linkages and Sustainable Regional Development: The Role of Entrepreneurs in Linking Peripheries and Centers. Sustainability 8(8), pp. 745.

Merkl A. (2007), Oberösterreich - Das führende Industrie-, Export- und Technologiebundesland Österreichs. Online verfügbar unter <https://shop.arl-net.de/media/direct/pdf/am_334. pdf $>$

MiöRnER J., TRIPPL M. (2017), Paving the way for new regional industrial paths: actors and modes of change in Scania's games industry. European Planning Studies 25(3), pp. 481-497.

Pike A., Rodríguez-Pose A., Tomaney J. (2017), Shifting horizons in local and regional development. Regional Studies 51(1), pp. 46-57.

Rodríguez-Pose A. (2018), The revenge of the places that don't matter (and what to do about it). Cambridge Journal of Regions, Economy and Society 11(1), pp. 189-209.

Sill T. (2019), Große Kluft mit vielen Vorurteilen. ORF, 08.04.2019. Online verfügbar unter $<$ https://orf.at/stories/3117491/>

Sotarauta M. (2010), Regional development and regional networks: The role of regional development officers in Finland. European Urban and Regional Studies 17(4), pp. 387-400. 
